\title{
Effect of Preoperative Nasal Retainer on Nasal Growth in Patients with Bilateral Incomplete Cleft Lip: A 3-Year Follow-Up Study
}

\author{
Young Chul Kim, Woo Shik Jeong, Tae Suk Oh, Jong Woo Choi, Kyung S. Koh \\ Department of Plastic and Reconstructive Surgery, Asan Medical Center, University of Ulsan College of Medicine, Seoul, Korea
}

Background The purpose of this study was to evaluate changes in nasal growth after the implementation of a preoperative nasal retainer in patients with bilateral incomplete cleft lip. Methods Twenty-six infants with bilateral incomplete cleft lip and cleft palate were included in the study. A preoperative nasal retainer was applied in 5 patients from the time of birth to 2.6-3.5 months before primary cheiloplasty. Twenty-one patients who were treated without a preoperative nasal retainer were placed in the control group. Standard frontal, basal, and lateral view photographs were taken 3 weeks before cheiloplasty, immediately after cheiloplasty, and at the 1- and 3-year postoperative follow-up visits. The columella and nasal growth ratio and nasolabial angle were indirectly measured using photographic anthropometry. Results The ratio of columella length to nasal tip protrusion significantly increased after the implementation of a preoperative nasal retainer compared to the control group for up to 3 years postoperatively $(\mathrm{P}<0.01$ for all time points). The ratios of nasal width to facial width, nasal width to intercanthal distance, columellar width to nasal width, and the nasolabial angle, for the two groups were not significantly different at any time point.

Conclusions Implementation of a preoperative nasal retainer provided significant advantages for achieving columellar elongation for up to 3 years postoperatively. It is a simple, reasonable option for correcting nostril shape, preventing deformities, and guiding development of facial structures.

Keywords Nose / Anthropometry / Cleft lip
Correspondence: Kyung S. Koh Department of Plastic and Reconstructive Surgery, University of Ulsan College of Medicine, Asan Medical Center, 88 Olympic-ro 43-gil, Songpa-gu, Seoul 05505, Korea

Tel: +82-2-3010-3603

Fax: +82-2-476-7471

E-mail: kskoh@amc.seoul.kr

Received: 6 Mar 2017 • Revised: 30 May 2017 Accepted: 4 Jul 2017

pISSN: 2234-6163 • elSSN: 2234-6171 • https://doi.org/10.5999/aps.2017.44.5.400 • Arch Plast Surg 2017;44:400-406

No potential conflict of interest relevant to this article was reported.

\section{INTRODUCTION}

Infants born with bilateral cleft lip are characterized by having a shortened columella and a protruding and elevated premaxilla, which results in the nose having an abnormal appearance. Although patients with bilateral complete cleft lip have a characteristic nasal deformity, those with an incomplete cleft lip have a nasal appearance that ranges from near normal to the characteristic deformity. In North America, according to one study, 36\% of surgeons routinely performed primary nasal repair to correct incomplete bilateral clefts, whereas $39 \%$ performed primary nasal repair only if deemed necessary [1].

Preoperative expansion of the nasal lining using preoperative nasal molding allows the deformed cartilage to be corrected at 
the time of primary repair and facilitates improved outcomes. The concept of tissue expansion was devised by Matsuo et al. [2], in a study in which they showed that alar cartilage is correctable using a nasal retainer in the early neonatal period. In 1998, Cutting et al. [3] developed a bilateral cleft lip and cleft palate nasal repair technique based on nonsurgical, lower-lateral cartilage molding and columellar elongation. Although several previous studies have reported the quantitative changes in nasal morphology following preoperative nasoalveolar molding, most of these were indicated for patients with bilateral complete cleft lip [4-7]. Relatively little is known about the effect of preoperative expansion of the nasal lining in patients with bilateral incomplete cleft lip.

In our experience, using a preoperative nasal retainer in patients with bilateral incomplete cleft lip is a simple and relatively inexpensive means of improving nasal morphology. The aim of this study was to evaluate the progressive changes in nasal growth following the application of a preoperative nasal retainer in patients with a bilateral incomplete cleft lip. We quantitatively analyzed the growth-related changes of nasal morphology up to 3 years postoperatively for patients treated with or without preoperative nasal retainers.

\section{METHODS}

We performed a longitudinal, retrospective review of 26 consecutive patients that were treated at our institution from 2002 through 2011. The criteria for inclusion were as follows: (1) incomplete bilateral cleft lip; (2) no other craniofacial malformation; (3) primary cheiloplasty and rhinoplasty performed by a single surgeon; and (4) patients with a follow-up period of at least 3 years postoperatively, with complete medical records and clinical photographs. Patients who showed significant asymmetry with a contralateral lesser form of cleft lip were excluded. Associated clefting of the alveolar ridge and secondary palate were noted.

In order to analyze the efficacy of the preoperative nasal retainer, patients were divided into 2 groups, based on whether or not they were treated with a preoperative nasal retainer. In total, 5 patients who were treated with a preoperative nasal retainer were included in the study group and 21 patients who were treated without a preoperative nasal retainer were included in the control group.

\section{Preoperative nasal retainer and lip taping}

Nasal retainer was applied immediately after the birth of an infant with a cleft-lip nasal deformity and was retained until primary cheiloplasty was performed. Because the nasal retainer

\section{Fig. 1. Preoperative nasal retainer and lip taping}

$(A, B) A$ preoperative nasal retainer and lip taping were applied immediately after birth. A silicone nasal conformer (Koken Co., Tokyo, Japan), number 1 size, was applied to this infant. The lateral rim was trimmed to the appropriate size. Lip taping was used to apply constant pressure on the maxilla and to retract the prolabium and approximate the cleft lip.

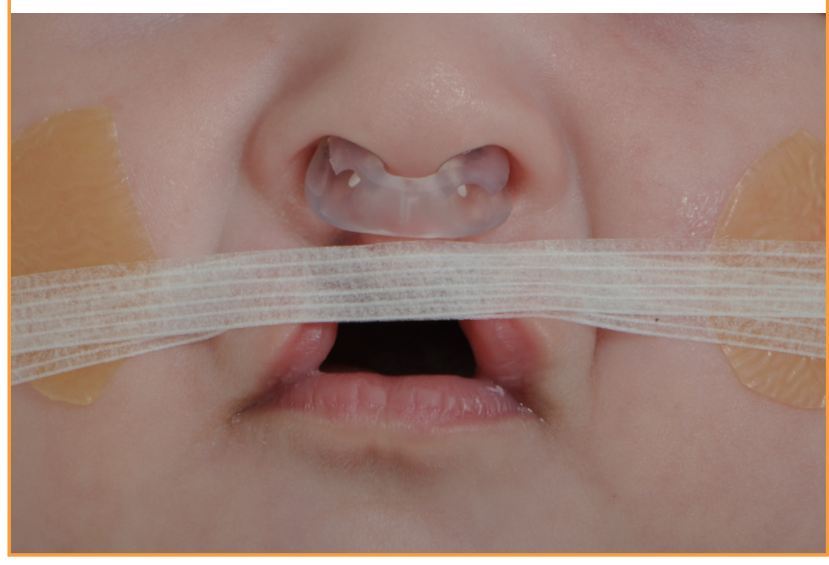

needs to be supported by the nostril sill, this technique could be used preoperatively in patients with bilateral incomplete cleft lip. A silicone nasal conformer (Koken Co., Tokyo, Japan) of appropriate size was fixed preoperatively using half-inch Mefix adhesive tape (SCA Mölnlycke Ltd., Dunstable, UK), and 2 holes were created with a hole puncher to match the position of each nostril. A modified tape-lip adhesion procedure was used to apply constant pressure on the maxilla to retract the prolabium and approximate the cleft lip (Fig. 1).

\section{Primary cheiloplasty and rhinoplasty}

We developed a surgical method for incomplete bilateral cleft lip that minimizes the transverse scar on the nostril sill, the paring of skin flaps, and the numbers of incisions. This method is an adaptation of our previously published method for repairing incomplete unilateral cleft lips (Fig. 2) [8]. The senior author performed closed rhinoplasty in conjunction with the cheiloplasty. Because Korean patients characteristically have a low nasal projection, a satisfactory nasal configuration could be achieved without approximation of the alar cartilage. Dissection of the nose was performed through the lip incision, and no additional incision was made at the alar base or in the nose. Transfixation sutures and a cinching suture at the alar base were made. A silicone nasal conformer was maintained for at least 6 months postoperatively.

\section{Anthropometric analysis}

All measurements were performed independently by 2 plastic surgeons. For the evaluation of surgical results, images that satis- 


\section{Fig. 2. Skin flap design and rhinoplasty}

(A) Design for minimal paring of skin flaps for bilateral incomplete cleft lips. (B) Closed rhinoplasty with blunt nasal dissection was performed without additional incision at the alar base. Transfixation sutures and a cinching suture at the alar base were made.
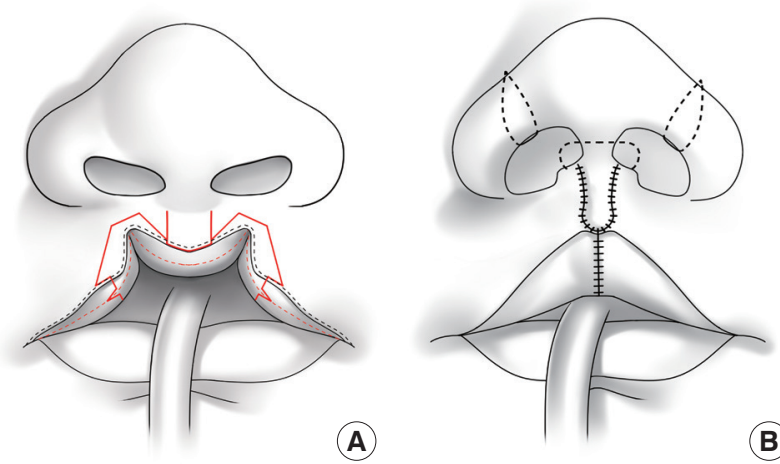

fied the following criteria were selected for evaluation: frontal view, both ears visible to indicate minimal rotation and least one nostril to indicate minimal tilt; basal view, nasal tip projected between medial canthi and eyebrows with no head rotation; and lateral view, lips in repose with one eye and columella visible to indicate minimal rotation.

Photographs were taken at 4 time points for each patient: 3 weeks preoperatively, immediately postoperatively, and 1 and 3 years postoperatively. The frontal view photographs were used to determine the columellar width, nasal width, intercanthal distance, and facial width. The basal view photographs were then used to determine the columellar length and nasal tip protrusion. Nasolabial angle was measured with the lateral view photographs.

The following parameters, as described by Farakas [9], were calculated from photogrammetric measurements (Fig. 3): nasal width to facial width ratio ( $\mathrm{Al}-\mathrm{Al} / \mathrm{Zy}-\mathrm{Zy})$, nasal width to intercanthal distance ratio (Al-Al/En-En), columellar length to nasal tip protrusion ratio (Sn-C/Sn-Prn), columellar width to nasal width ratio ( $\left.\mathrm{Sn}^{\prime}-\mathrm{Sn}^{\prime} / \mathrm{Al}-\mathrm{Al}\right)$, and nasolabial angle. Changes in columellar features and nasal growth were observed for both the study and control groups.

\section{Statistical analysis}

Statistical analysis was conducted using SPSS ver. 20 (IBM Corp., Armonk, NY, USA). A linear mixed model for repeated measures was used to test for differences in nasal features between the study and control groups over the study period using the photogrammetric data. The 4 time points for the implementation of a preoperative nasal retainer were included as covariates in the model. The differences between the groups at each time point and the differences between the 4 time points were

\section{Fig. 3. Anthropometric points}

Zygomatic prominence, zygion (Zy); alar convexity, alare (Al); medial canthus, endocanthion (En); subnasale $(\mathrm{Sn})$; nasal tip, pronasale (Prn); top of columella ( $\left.C^{\prime}\right)$; lateral border of columellar base $\left(S n^{\prime}\right)$; and upper midline vermilion-cutaneous junction, labiale superioris $(L s)$. The nasiolabial angle $(\alpha)$ was also measured.

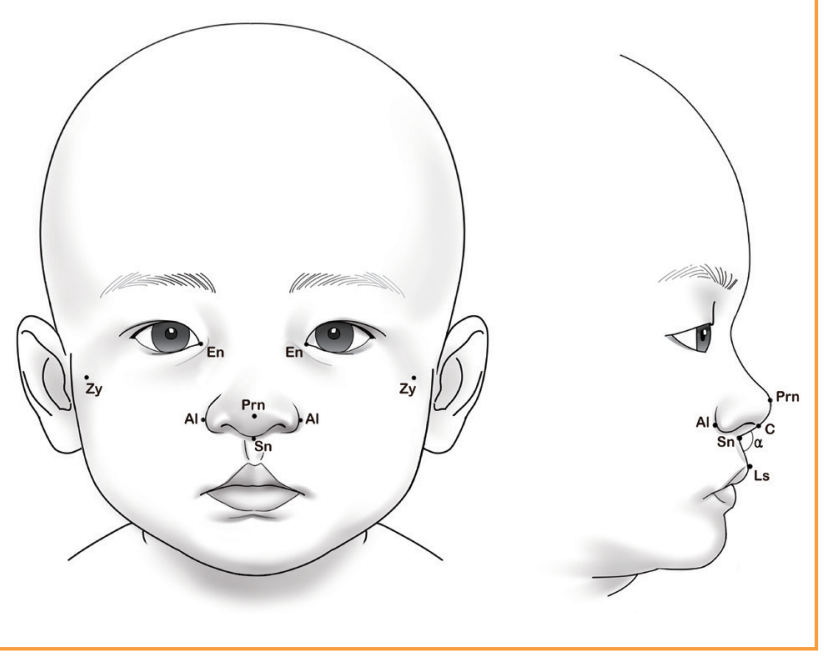

tested based on the corresponding contrasts of the parameters estimated in the mixed model.

Intraobserver and interobserver variability was measured to determine measurement reliability of randomly selected cases. For intraobserver variability, 10 different randomly selected photographs were measured twice, 1 week apart, by the same person. For interobserver variability, two 3-year postoperative photographs of the same infant were measured from 10 randomly selected patients. Intraclass correlation coefficients were used to determine intraobserver and interobserver reliability.

\section{RESULTS}

Of 26 patients with bilateral incomplete cleft lip, 10 were female and 16 were male. The mean age at the time of surgery was 12.7 weeks (range, 11.1-21.9 weeks). The preoperative nasal retainers were applied immediately after birth, and the treatment duration was from 80 to 106 days (mean, 91 days). Both cleft alveolus and cleft palate were observed in 12 patients (46.1\%) and cleft alveolus without cleft palate was observed in 11 patients (42.3\%). Only 3 out of the 26 patients (11.5\%) had an isolated bilateral cleft. A highly significant intraobserver correlation $(\mathrm{r}=0.983, \mathrm{P}<0.001)$ was found for repetitive measurements, as was the case for interobserver variability $(\mathrm{r}=0.973, \mathrm{P}<0.001)$ between the photographs. Serial anthropometric measurements were analyzed for all patients (Fig. 4).

Linear mixed model analyses showed no significant interaction between the groups and the time points. Therefore, only 


\section{Fig. 4. Serial photographs of a patient with a retainer}

Serial photographs of one of the patients who received a preoperative nasal retainer until the 3-year follow-up: (A) immediately after birth (before the implementation of nasal retainer); (B) 3 weeks preoperatively; (C) just before surgery; (D) just after surgery; (E) 1 year postoperatively; and (F) 3 years postoperatively.
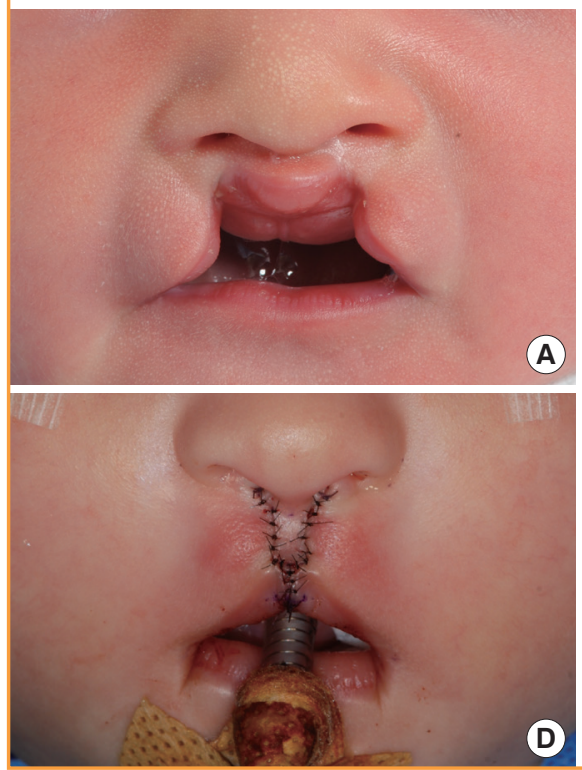

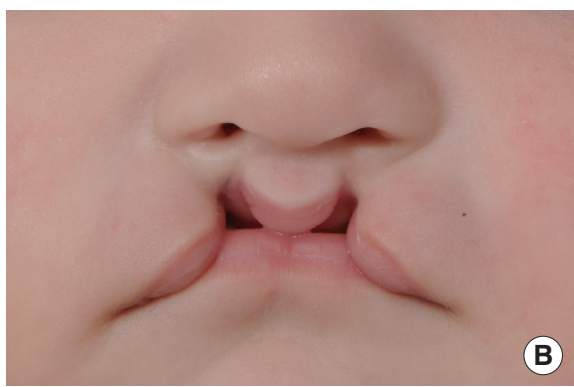

B

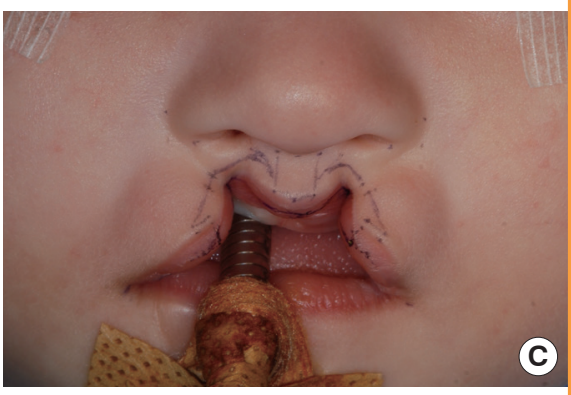

(E)

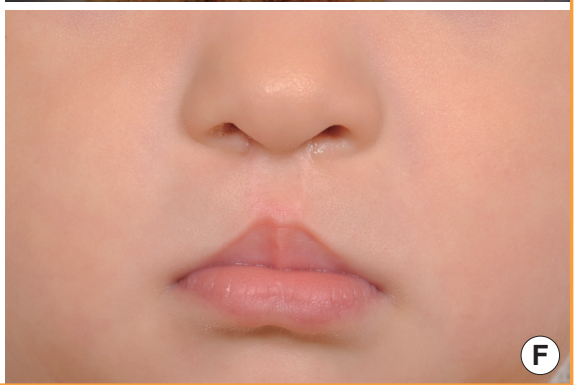

the group effect is presented here. The nasal width to facial width ratio ( $\mathrm{Al}-\mathrm{Al} / \mathrm{Zy}-\mathrm{Zy})$, the nasal width to intercanthal distance ratio (Al-Al/En-En), and the columellar width to nasal width ratio $\left(\mathrm{Sn}^{\prime}-\mathrm{Sn}^{\prime} / \mathrm{Al}-\mathrm{Al}\right)$ for the 2 groups were not significantly different at any time point. Nasal width initially decreased postoperatively and then gradually grew in parallel with facial growth over the 3-year postoperative period. The nasal width and intercanthal distance ratio $(1: 1)$ indicated they remained similar at 1 and 3 years postoperatively. Additionally, columellar width and nasal width increased similarly over the 3-year postoperative period. The ratio of columellar length and nasal tip protrusion was significantly higher $(\mathrm{P}=0.005)$ for the preoperative nasal retainer group than the control group for all time points. Both values increased slightly postoperatively and then gradually decreased over the 3-year postoperative period as the nose continued to grow. The nasolabial angle of the two groups was not significantly different for all time points. It increased postoperatively and remained similar up to 3 years postoperatively. The results of the anthropometric analysis are summarized in Fig. 5.

\section{DISCUSSION}

A number of studies have documented the effect of preoperative expansion of the nasal lining using the nasal molding technique [3-7]. However, most of these procedures were performed in patients with bilateral complete cleft lip and cleft palate. Rela- tively little attention has been paid to patients with bilateral incomplete cleft lip. Mulliken et al. [10] affirmed that nasal deformities were less obvious in cases with an incomplete rather than complete cleft lip. Considering the results of this study, the nasal molding technique seems to be beneficial for achieving columellar growth following the application of a preoperative nasal retainer in patients with an incomplete cleft lip for up to 3 years postoperatively.

Although preoperative nasoalveolar molding has been routinely performed in patients with bilateral cleft lip and cleft palate, it is a time-consuming process and creates a financial burden on the patients. Moreover, compliance issues are of particular concern and can lead to treatment failure [11]. We suggested that the application of a preoperative retainer with lip taping could overcome some of these obstacles encounterd in the application of preoperative nasoalveolar molding. In our experience, a nasal retainer is a simple and relatively inexpensive alternative option that can be used to improve the nasal morphology of patients with bilateral incomplete cleft lip and cleft palate.

The columella length and nasal tip protrusion increased at different rates postoperatively. Liou et al. [7] reported that the columella remained at a similar length 3 years after presurgical nasoalveolar molding and primary cheiloplasty. Although our results showed a similar tendency for a relative relapse in terms of columella length compared to nasal tip height in both groups, we found that the application of a preoperative nasal retainer significantly lengthened the columella for up to 3 years postop- 


\section{Fig. 5. Anthropometric results}

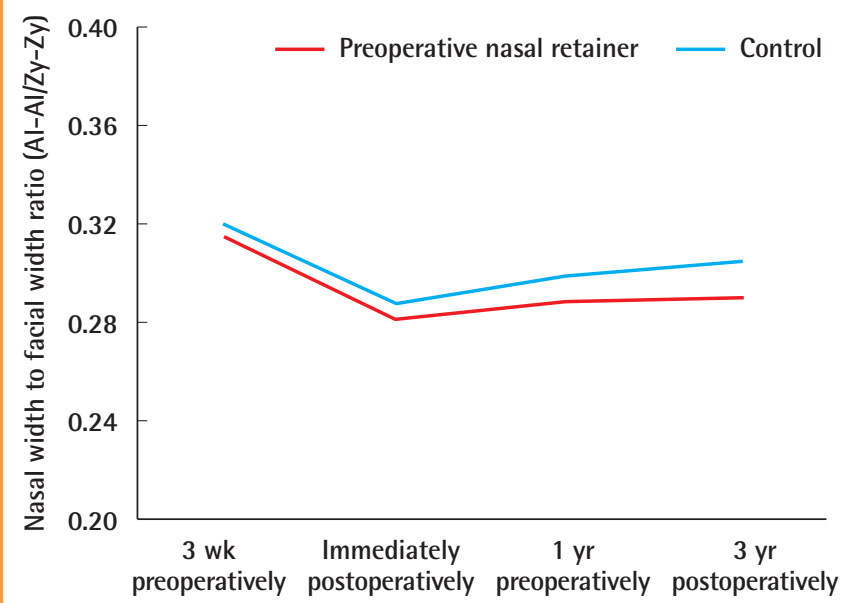

Time point

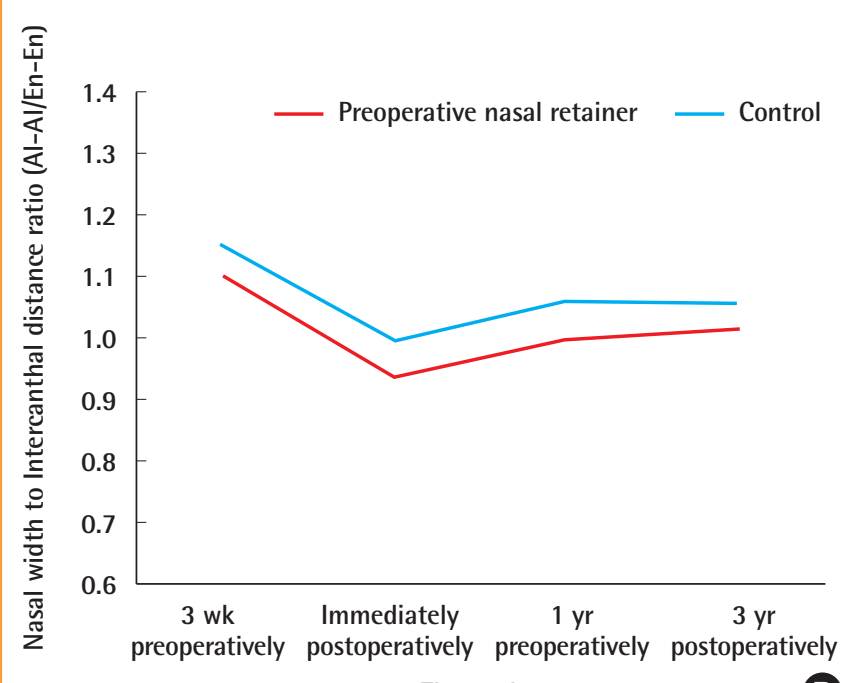

Time point

B
Results of anthropometry in patients with bilateral incomplete cleft lip with or without preoperative nasal retainer: (A) Nasal width to facial width ratio (Al-Al/Zy-Zy); (B) Nasal width to intercanthal distance ratio (Al-Al/En-En); (C) Columellar length to nasal tip protrusion ratio (Sn-C/Sn-Prn); ${ }^{a}$ Statistical significance. (D) Columellar width to nasal width ratio (Sn'-Sn'/Al-Al); (E) Nasolabial angle ( ${ }^{\circ}$. The ratio of columellar length and nasal tip protrusion was significantly higher in the preoperative nasal retainer group for all time points. Al, alare; Zy, zygion; En, endocanthion; Sn, subnasale; C, columella; Prn, pronasale; $S n$ ', lateral border of columellar base.
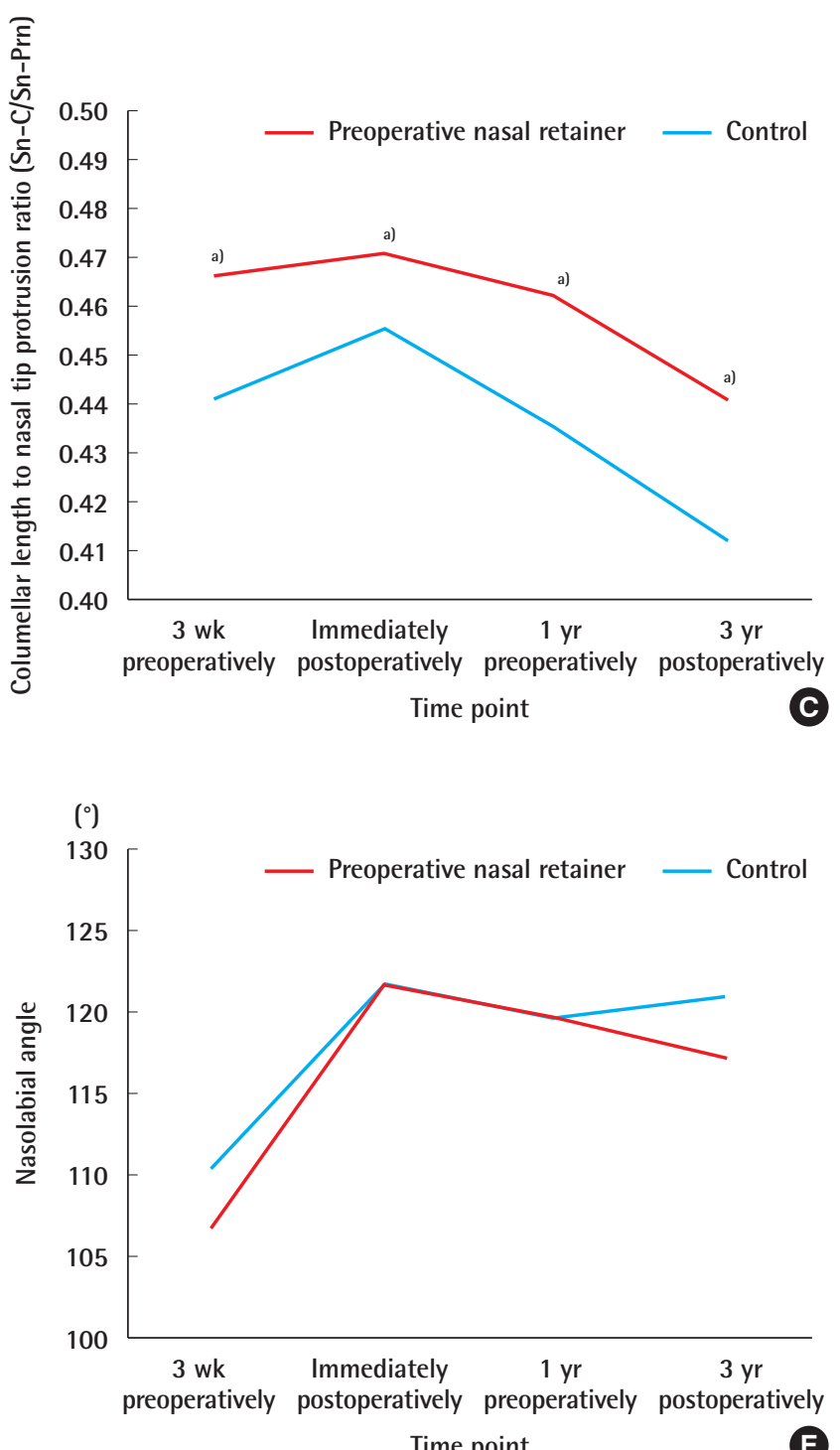
eratively.

Regarding the transverse growth of the nose, the preoperative nasal retainers had little impact on columellar and nasal width. Nasal width was initially set by the surgeon at the time of primary cheiloplasty and then gradually increased to reach the intercanthal distance. In terms of aesthetics, a nasal width that corresponds to the intercanthal distance can provide an attractive appearance; it also implies that the alar width was properly set at the time of primary reconstruction [12]. In our experience, dissection just below the alar base and cinching the sutures play an important role in preventing alar widening. However, this needs to be confirmed with additional, long-term follow-up.

Nasal retainers alone are not sufficient to successfully elongate the columella. We have found that elevating the nostril to elongate the columella without pulling down the prolabium simultaneously results in loss of tensile strength. The addition of lip taping counteracts the effect of the nasal retainer by placing constant pressure on the prolabium. It is imperative, as Cutting stated, that a "saddle" be placed at the lip-columella junction to define the separation between lip and columella and allow the columella to be expanded along an anterior vector while tape is used to stretch the prolabium inferiorly [3]. The parents of the patients adjusted the lip taping to maintain the tension and also cleaned the retainer daily.

Garfinkle et al. [6] stated that the nasoalveolar molding should be combined with primary retrograde nasal reconstruction to prevent lower lateral cartilage relapse and to improve tip shape by removing the fibrofatty tissue that is interposed between the widely separated dome cartilages. Chang et al. [13] also noted that without surgical repositioning of the alar cartilage, some relapse might occur even after the implementation of nasoalveolar molding therapy. In this study, however, we performed closed rhinoplasty without any incision on the nostril. In our experience, in Korean patients with a characteristically low nasal projection, a satisfactory nasal configuration can be achieved through conservative management without approximation of the alar cartilage, simply by dissection and redraping through the lip incision, transfixation, and alar-base cinching sutures, along with the use of silicone nasal conformers. Most of our patients with a bilateral incomplete cleft lip and cleft palate did not require secondary rhinoplasty until they were of preschool age.

There are some limitations to this study. First, the sample size of the preoperative nasal retainer group is too small to represent the full set of characteristics of this group. Future studies that include a large group of patients will be needed to confirm our findings. Second, the control group should represent normative anthropometric values; however, there is not an accepted reference guide for Korean children similar to the Farkas data set, which is used for Caucasians. Although it is not an entirely fair comparison due to lack of referable data for normal Korean children, comparing the retainer group to those without a retainer implies that the nasal configuration of the retainer group is superior to that of the control group in terms of achieving columellar elongation. Third, we could not describe the original, pre-treatment measurements for the 2 groups, due to a lack of photographs and inconsistencies in the patients' first visit to the clinic. Because the decision to perform a preoperative nasal retainer was not made randomly, patients may have been more likely to be in the retainer group when they had a relatively lowprofile nose with a short columella. This selection bias, however, may support our conclusion that the increase in columellar length in the preoperative retainer group reflects the effectiveness of the treatment at least by the 3-year follow-up. Finally, the present study relied on photogrammetric measurements, which is one of the most accessible methods for recording treatment progress, as it is noninvasive and is acceptable to both the patients and their parents. To minimize errors when using this technique, all photographs were taken by the same photographer under the same conditions, and the measurements were evaluated as ratios. Furthermore, 3-dimensional photographic scans may be used in the future to obtain more accurate measurements.

Consequently, we have quantitatively shown that preoperative nasal retainers in patients with a bilateral incomplete cleft lip provided significant advantages for achieving columellar elongation for up to 3 years postoperatively. It is a simple, reasonable option for correcting nostril shape, preventing deformities, and guiding development of the facial structures.

\section{PATIENT CONSENT}

The patient provided written informed consent for the publication and the use of their images.

\section{REFERENCES}

1. Tan SP, Greene AK, Mulliken JB. Current surgical management of bilateral cleft lip in North America. Plast Reconstr Surg 2012;129:1347-55.

2. Matsuo K, Hirose T, Tomono T, et al. Nonsurgical correction of congenital auricular deformities in the early neonate: a preliminary report. Plast Reconstr Surg 1984;73:38-51.

3. Cutting C, Grayson B, Brecht L, et al. Presurgical columellar elongation and primary retrograde nasal reconstruction in one-stage bilateral cleft lip and nose repair. Plast Reconstr Surg 1998;101:630-9. 
4. Lee CT, Garfinkle JS, Warren SM, et al. Nasoalveolar molding improves appearance of children with bilateral cleft lipcleft palate. Plast Reconstr Surg 2008;122:1131-7.

5. Spengler AL, Chavarria C, Teichgraeber JF, et al. Presurgical nasoalveolar molding therapy for the treatment of bilateral cleft lip and palate: a preliminary study. Cleft Palate Craniofac J 2006;43:321-8.

6. Garfinkle JS, King TW, Grayson BH, et al. A 12-year anthropometric evaluation of the nose in bilateral cleft lip-cleft palate patients following nasoalveolar molding and cutting bilateral cleft lip and nose reconstruction. Plast Reconstr Surg 2011;127:1659-67.

7. Liou EJ, Subramanian M, Chen PK. Progressive changes of columella length and nasal growth after nasoalveolar molding in bilateral cleft patients: a 3-year follow-up study. Plast Reconstr Surg 2007;119:642-8.

8. Koh KS, Choi JW, Kim H. Minimal paring of skin flaps for primary repair of incomplete unilateral cleft lip. Plast Recon- str Surg 2008;121:1382-5.

9. Farakas LG. Anthropometry of the head and face. 2 nd ed. New York: Raven Press; 1994.

10. Mulliken JB, Kim DC. Repair of bilateral incomplete cleft lip: techniques and outcomes. Plast Reconstr Surg 2013; 132:923-32.

11. Vyas HJ, Sharma SM, Shetty V. Levy-Bercowski D, Abreu A, DeLeon E, Looney S, Stockstill J, Weiler M, Santiago PE. Complications and solutions in presurgical nasoalveolar molding therapy. Cleft Palate Craniofac J. 2009;46:521-8. Cleft Palate Craniofac J 2012;49:766.

12. Kim SH, Whang E, Choi HG, et al. Analysis of the midface, focusing on the nose: an anthropometric study in young Koreans. J Craniofac Surg 2010;21:1941-4.

13. Chang CS, Liao YF, Wallace CG, et al. Long-term comparison of the results of four techniques used for bilateral cleft nose repair: a single surgeon's experience. Plast Reconstr Surg 2014;134:926e-936e. 